\title{
COMMUNICATION
}

\section{A propos d'un cas de lymphome osseux primitif maxillaire}

\section{Provost $\mathrm{M}^{1,2}$, Morel $\mathrm{V}^{3}$, Geneviève $\mathrm{H}^{4}$, Descroix $\mathrm{V}^{1,5}$, Lescailles $\mathrm{G}^{1,5,6}$}

1. Service d'Odontologie, Groupe Hospitalier Pitié-Salpêtrière, Assistance Publique-Hôpitaux de Paris (AP-HP) - Paris

2. Faculté de chirurgie dentaire Paris Descartes, département de médecine buccale et chirurgie buccale, Montrouge, France

3. Service d'Hématologie, Groupe Hospitalier Pitié-Salpêtrière, Assistance Publique-Hôpitaux de Paris (AP-HP) - Paris

Sorbonne Universités UPMC - Paris 06

4. Service d'Anatomopathologie, Groupe Hospitalier Pitié-Salpêtrière, Assistance Publique-Hôpitaux de Paris (AP-HP) - Paris Sorbonne Universités UPMC - Paris 06

5. Université Paris Diderot - Sorbonne Paris Cite - Paris 07

6. Centre d'Immunologie et Maladies Infectieuses (CIMI-Paris), UMR-S INSERM U1135, CNRS ERL 8255 , Sorbonne Universités, UPMC - Paris 06

\section{Introduction}

Le lymphome osseux primitif (LOP) est une entité rare (moins de 1\% des lymphomes non hodgkiniens), atteignant principalement les hommes entre 45 et 60 ans. II se présente sous la forme de lésion osseuse unique ou multiple, en association ou non à des atteintes ganglionnaires régionales. Les symptômes d'appel sont souvent non spécifiques et souvent tardifs, de type douleur (80 à $95 \%$ des cas) ou tuméfaction en lien avec le développement de la masse tumorale ( 30 à $40 \%$ des cas).

\section{Patient}

II s'agissait d'un patient âgé de 26 ans sans antécédents médicaux ni altération de l'état général, rapportant des douleurs maxillaires droites évoluant depuis environ un an, associées à une tuméfaction jugale intermittente et résolutive sous antibiotiques et anti-inflammatoires. L'examen clinique exobuccal ne retrouvait aucune particularité. L'examen endobuccal mettait en évidence une mobilité 2 de 16 et des douleurs gingivales dans le même secteur. L'orthopantomogramme révélait une trame osseuse maxillaire droite inhomogène, aux contours flous. Sur le CBCT réalisé un an auparavant par un confrère, on pouvait noter sur les coupes axiales, un remaniement de la trame osseuse alvéolaire, avec un aspect hétérogène, accompagné d'un élargissement desmodontal du secteur prémolo-molaire droit plus marqué sur la 16 ; un diagnostic de parodontite localisée avait alors été posé.

Compte tenu de l'ensemble des éléments et du caractère inhomogène osseux, une biopsie a alors été réalisée par abord vestibulaire permettant de diagnostiquer un lymphome de type $\mathrm{B}$ diffus à grandes cellules centro-germinatif. Un bilan d'extension comprenant un TDM injecté complété par un TEP-scanner permettait de visualiser une volumineuse masse tissulaire envahissant le sinus adjacent, les cornets ainsi que l'os maxillaire gauche par contiguïté, une adénomégalie de la loge thymique d'allure nécrotique, ce qui concluait à un lymphome agressif localisé et extranodal. Sur le plan biologique, on notait une légère lymphocytopénie $\left(1.29 \times 10^{*} 9 / \mathrm{L}\right)$, un LDH normal, et des sérologies négatives. Les résultats de la biopsie ostéo-médullaire étaient normaux et l'analyse du LCR avait permis d'écarter une atteinte neuro-méningée.

La prise en charge hématologique a consisté en six cures d'immuno-chimiothérapie par R-CHOP (Rituximab, Doxorubicine, Vincristine, Ciclophosphamide, Cortancyl) qui ont été bien tolérées. Les examens radiologiques de contrôle ainsi que la consultation d'hématologie à un an concluaient à une rémission complète.

Au niveau local un nouveau CBCT permettait d'objectiver une normalisation de l'aspect osseux du maxillaire, avec absence d'élargissement desmodontal du secteur précédemment concerné.

(C) The authors, published by EDP Sciences. This is an Open Access article distributed under the terms of the Creative Commons Attribution License 4.0 (http://creativecommons.org/licenses/by/4.0/). 
$65^{\text {ème }}$ Congrès de la SFCO

\section{Discussion}

Les lymphomes primaires osseux de la sphère oro-faciale sont rares et il s'agit le plus fréquemment de lymphomes diffus à grandes cellules B (LDGCB). II se distingue des lymphomes osseux secondaires, qui correspondent quant à eux à des atteintes osseuses au cours d'un lymphome disséminé avec d'autres signes d'appel plus caractéristiques. Le but de ce cas clinique est de souligner l'importance de la biopsie osseuse qui doit être systématique dans un contexte de douleur inexpliquée et de modification de la trame osseuse associée.

prvst.math@gmail.com

\section{Bibliographie}

Messina C. et al. Primary and secondary bone lymphomas. Cancer treatment reviews, 41(3), 235-246.

Bosly A et al. Lymphomes di us a grandes cellules B. EMC, Hématologie, 13-01-A-60, 2007

Renard, N. et al. Lymphomes malins non hodgkiniens buccaux: A propos de 18 cas. Méd Bucc Chir Bucc, 21(2), 77-83. 